\title{
Capillary Gripping and Self-alignment: A Route Towards Autonomous Heterogeneous Assembly
}

\author{
G. Arutinov, M. Mastrangeli, G. van Heck, \\ P. Lambert, J. M. J. den Toonder, A. Dietzel, E. C. P. Smits
}

\begin{abstract}
We present a pick-and-place approach driven by capillarity for highly precise and cost-effective assembly of mesoscopic components onto structured substrates. Based on competing liquid bridges, the technology seamlessly combines programmable capillary grasping, handling and passive releasing with capillary self-alignment of components onto pre-patterned assembly sites. The performance of the capillary gripper is illustrated by comparing the measured lifting capillary forces with those predicted by a hydrostatic model of the liquid meniscus. Two component release strategies, based on either axial or shear capillary forces, are discussed and experimentally validated. The releaseand-assembly process developed for a continuously moving assembly substrate provides a roll-to-roll-compatible technology for high-resolution and high-throughput component assembly.
\end{abstract}

Index Terms - Capillary gripper, liquid bridge, foil-to-foil integration, self-alignment, modeling, moving web.

\section{INTRODUCTION}

$\mathrm{F}$ OLLOWING a current consumer trend, the demand for high-performance and highly-integrated multifunctional systems is expected to further increase in the coming years [1]. Addressing this industrial need eminently requires cost- and time-effective integration of micro- and nanoelectronic components-performing complementary functions such as e.g. sensing and actuation, transceiving, power management-onto functional circuitry. Most of these components, however, are subject to mutually conflicting fabrication requirements and processing constraints. This makes purely monolithic integration demanding [2]. Heterogeneous integration onto a carrier substrate is therefore regarded as a promising alternative [3]. Advanced devices benefiting from hybrid integration of separately manufactured subsystems include, among others, radio-frequency communication units [4], addressable sensor arrays, and light emitting diodes [5].

This work was funded through Marie Curie ITN FlexSmell project $\mathrm{N}^{\circ} 238454$ and by the Interuniversity Attraction Poles Programme (IAP 7/38 MicroMAST) initiated by the Belgian Science Policy Office.

G. Arutinov, E. C. P. Smits, G and van Heck are with the Holst Centre/TNO, 5656 AE, Eindhoven, The Netherlands (e-mail: gari.arutinov@gmail.com; edsger.smits@tno.nl; gert.vanheck@tno.nl).

M. Mastrangeli and P. Lambert are with the Department of Bio, Electro And Mechanical Systems (BEAMS), École Polytechnique, Université Libre de Bruxelles, 1050 Bruxelles, Belgium (e-mail: massimo.mastrangeli@ulb.ac.be; pierre.lambert@ulb.ac.be).

J. M. J. den Toonder is with the Department of Mechanical Engineering, Eindhoven University of Technology, 5600 MB Eindhoven, The Netherlands (e-mail: j.m.j.d.toonder@tue.nl)

A. Dietzel is with the Institute of Microtechnology, Technical University of Braunschweig, 38124 Braunschweig, Germany (e-mail: a.dietzel@tubraunschweig.de).

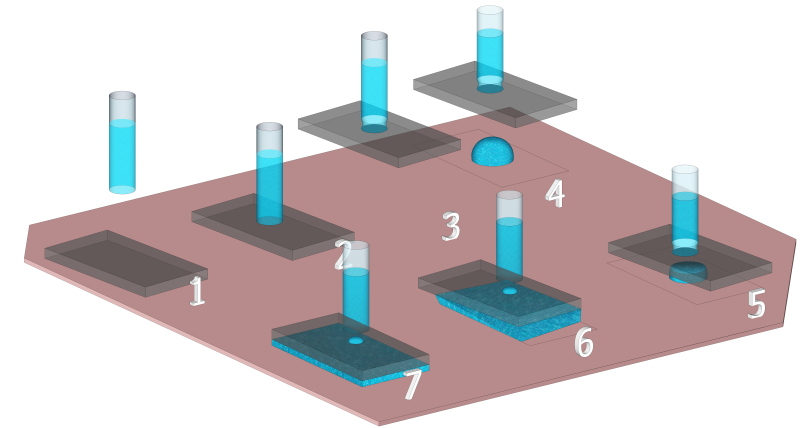

Fig. 1. Sketch of the sequential process steps for component-to-substrate integration (exemplified as foil die-to-foil carrier integration in this work) by capillary gripping and self-alignment: (1) water-filled capillary tube (i.e. the capillary gripper) approaching the foil die, (2) formation of the capillary-die liquid bridge by sealing of the bottom end of the capillary, (3) vertical lifting of the foil die by retraction of the capillary, (4) patterning of the assembly site on the carrier substrate, and controlled water dispensing within the inner confining ring, (5) coarse pre-alignment of the foil die over the site, and formation of the die-carrier liquid bridge, (6) release of the foil die from the gripper, and (7) constrained self-alignment on the assembly site.

Assembly of components onto pre-processed substrates such as printed circuit boards or back-end-of-line processed silicon wafers relies on the accurate registration of the components onto predefined assembly sites. The operation involves fetching, grasping, positioning and releasing components. This consistent set of actions is commonly performed by robotic effectors equipped with force feedback and gripping tools [6] to specifically handle components of centimetric down to micrometric sizes [7]. A variety of gripping principles and methods have been demonstrated [8-11], including contactless handling techniques [12-14]. Nevertheless, most conventional mechanical grippers operate in direct contact mode, and in absence of compliance the force they exert may damage delicate components [8]. By subsuming compliant component grasping, component self-centering and favorable force versus size scaling [15], capillary gripping represents a non-intrusive manipulation technique of choice for a large range of component assembly tasks.

A capillary gripper is a robotic end effector handling a liquid droplet at its active extremity. The gripper touches an approaching component indirectly through the droplet. Upon contact, the droplet establishes a liquid bridge between gripper and component. The compliance of the droplet avoids imparting excessive shocks to the components; and by a tailored wetting action, the droplet can reliably grasp objects even with rough and warped surfaces. The axial force ensuing from confined liquid bridges allows lifting components weighting up to tens of mg [6]. Even for relatively high accelerations, 


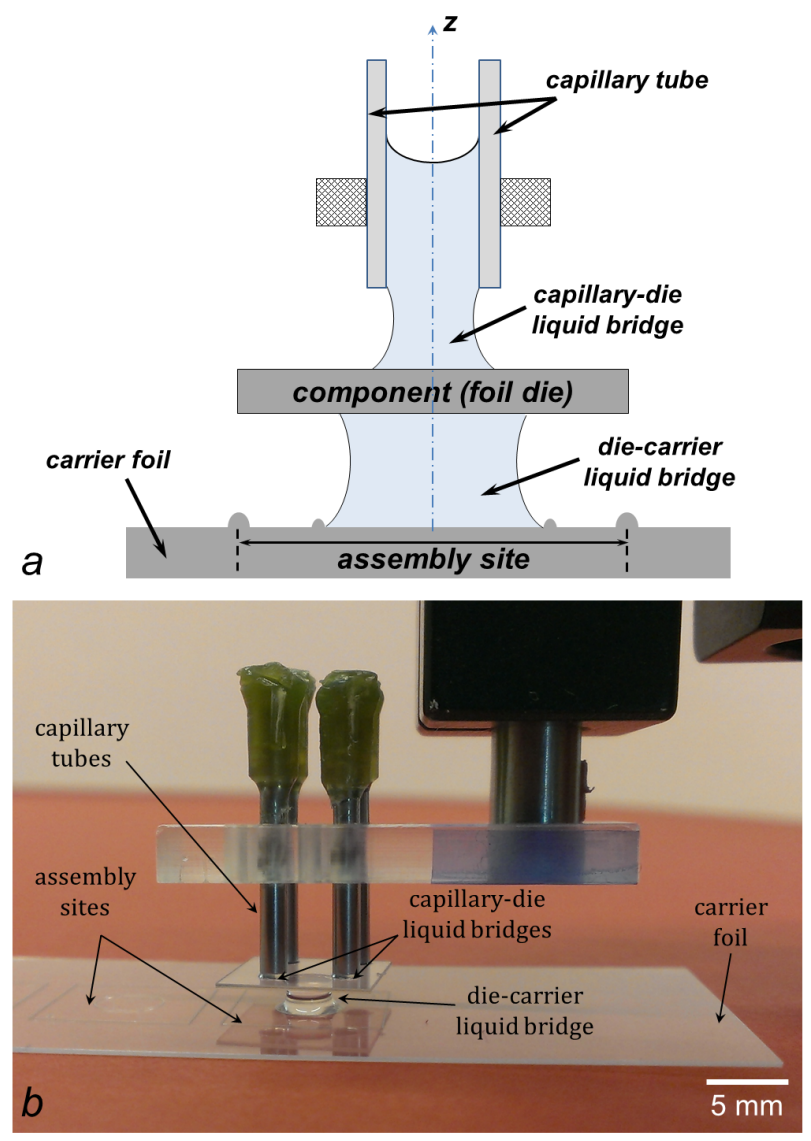

Fig. 2. Competing liquid bridges for capillary component gripping and passive release to a substrate. (a) A component held between the capillary-die (top) and die-carrier (bottom) liquid bridges (sketch not to scale). (b) Implementation of the capillary system for foil-to-foil integration.

and in absence of additional constraints for component release, the capillary lifting force can prevail over the apparent component weight.

Concerning component release, the variety of strategies available can be generally categorized as passive or active [6]. Passive release strategies exploit environmental conditions like surface features to reduce adhesive forces between gripper and component [16-20]. Active release strategies employ specific gripper actions [10], notably the modification of gripper curvature [21], change of meniscus pressure by gas injection [22]. We previously reported an integration process based on topographical surface structuring via laser patterning for accurate capillary self-alignment of plastic electronic devices [23]. In that robust but manual process, foil dies were coarsely superposed onto water-coated assembly sites by means of vacuum tweezers before accurately self-aligning through capillarity and shape matching.

In this paper, we introduce and characterize a componentto-substrate integration concept based on the seamless combination of capillarity-driven component gripping, passive release and self-alignment onto structured substrate sites. The process exploits competing liquid bridges [19-20] to autonomously align mesoscopic components onto pre-patterned substrates (Fig. 1). The gripper is composed of several capillaries, i.e. cylindrical liquid-filled tubes with both ends open and attached to a programmable and remotely-controlled motion stage (Fig. 2). The integration process only requires coarse alignment steps, both to bring the gripper into contact with the component and, subsequently, to bring the component in contact with a liquid droplet confined on the substrate. Furthermore, the combination of capillary gripping with a novel dynamic shear release technique enables an assembly process with superior throughput and accuracy, and opens a solid route towards autonomous and highly reliable roll-to-roll component integration.

\section{CAPILLARY GRIPPING AND SELF-ALIGNMENT}

\section{Experimental procedure}

The assembly process presented in this work (Fig. 1) relies on the consistent combination of individually well-mastered techniques: topographic surface structuring, capillary gripping, competing liquid bridges and capillary self-alignment [24]. Through the process, a generic target substrate can be efficiently populated with components of known footprint.

First the substrate is structured to support the self-alignment process. Two sets of trenches were laser-scribed in a $125 \mu \mathrm{m}-$ thick Polyethylene Naphthalate (PEN) foil substrate (Teonex ${ }^{\circledR}$ Q65FA, Teijin DuPont) [23] using a Coherent AVIA $355 \mathrm{~nm}$, $25 \mathrm{~ns}$ Nd:YAG laser source in combination with a galvanoscanner: deep, outer trenches defining the assembly sites; and shallower, inner circular trenches to precisely confine a single liquid droplet in the center of each site. Foil dies mimicking centimetric components were similarly laser-machined from transparent, $250 \mu \mathrm{m}$-thick PEN sheets to exactly match the geometry of the assembly sites. Joint markers were scribed onto the foil dies and the assembly sites to provide a quantitative measure of the alignment accuracy. Fabrication details can be found in a previous publication [23].

The assembly process starts by dispensing a water droplet within the inner trench of each assembly site. The confined droplet of known volume assumes thereby a predictable profile [23]. The wettability of the top surface of the foil dies was increased through an oxygen plasma treatment $(400 \mathrm{~W}$ for 1 $\mathrm{min}$ ) that decreased the static water contact angle (from $65^{\circ} \pm$ $3^{\circ}$ to $8^{\circ} \pm 2^{\circ}$ ) and improved adhesion with the gripper. The same surface treatment can similarly increase the forces exerted by the assembly substrate during capillary self-alignment [25]. This was however omitted, as it was found to inhibit droplet confinement and correct component registration [23].

Next, the capillaries of the gripper are filled with water. An open-ended capillary of millimetric diameter automatically fills up when one end is immersed in a reservoir of wetting liquid. Upon retraction from the water/air interface, a small droplet protrude from the bottom end of each capillary. By subsequently bringing the water-filled capillaries into contact with a component, the bottom ends of the capillaries are sealed, forcing the wetting of the component surface and the formation of the capillary-die liquid bridge (Fig. 2). The component can then be lifted upon vertical retraction (i.e. pull-off) of the gripper.

By bringing the foil die into contact with the droplet resting on an assembly site, a die-carrier liquid bridge is formed (Fig. 2 ). As the water spreads over the confining surfaces, the axial force exerted by the die-carrier liquid bridge gradually in- 

HERE TO EDIT) $<$

creases. When this force exceeds that of the competing capillary-die liquid bridge, the latter collapses releasing the foil die from the gripper. Henceforth the die self-aligns onto its matching assembly site driven by the constrained relaxation of the underlying liquid meniscus [26].

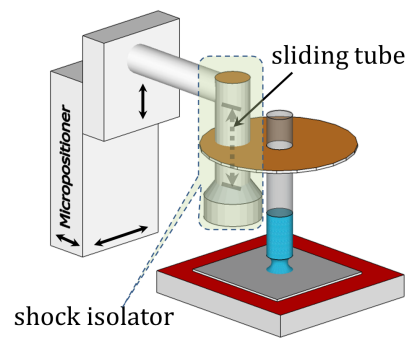

a
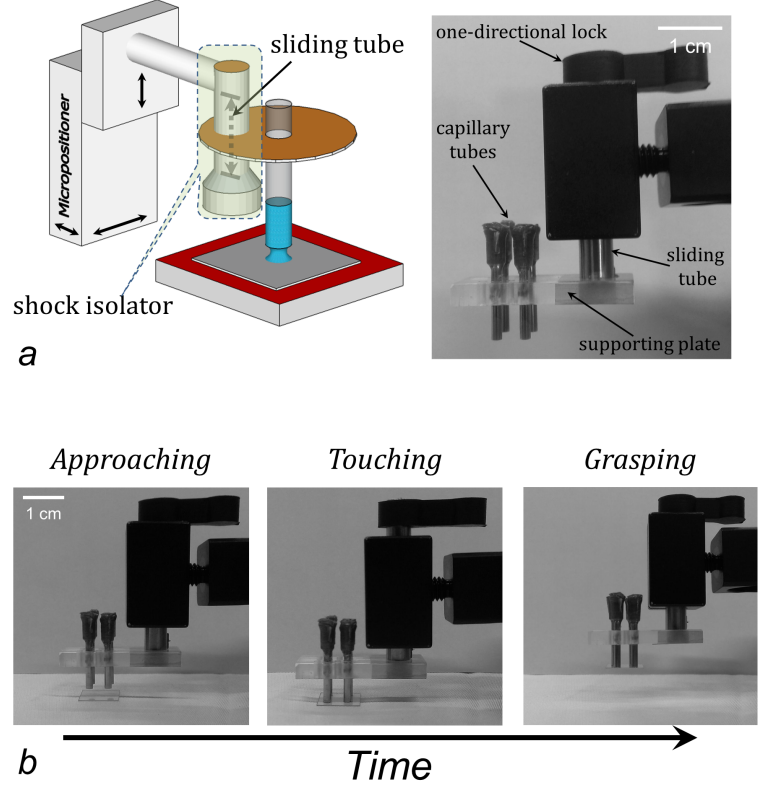

Fig. 3. (a) Schematic representation (not to scale) and side view of the capillary gripper with shock isolator lock used to reduce the impulsive forces exerted on components. (b) Timeframe showing the grasping sequence of the capillary gripper. The gripper approaches vertically the foil die (left), until it establishes contact with it and the shock isolator glides up (middle). Hence the foil die is grasped, the gripper is pulled off and the shock insulator glides down (right).

Contact of the foil die with the confined droplet could be initiated in various ways, each yielding a specific release method. In particular, either the substrate or the gripper can be in motion, and component release can be initiated either vertical$l y$ or horizontally (i.e. by lateral shear). In vertical release, the gripper lowers the component onto the droplet from above. Horizontal component release is conversely enacted as the gripper translates the component laterally and slides it into contact with the droplet at a controlled distance from the substrate. Both methods will be described in more details below.

\section{Realization of the capillary gripper}

The component capture range of a capillary tube is set by the vertical protrusion of the inner liquid from its bottom end. In the limit of submillimetric droplets, accurate positioning and/or force feedback are typically employed to avoid potentially damaging impacts between gripper and components. A prior gripper had a water-filled glass capillary whose pushpull action deformed the protruding water drop to change the working range of the gripper [27]. We developed a compliant capillary gripper by using a shock insulator lock (formed by a sliding piston, see Fig. 3). The capillary of the gripper is suspended by the shock insulator, which glides upwards when in contact with an object (Fig. 3a). In this way, the grasped component experiences the mechanical impact exerted solely by the cumulative weight of capillary and supporting piston mechanism, amounting to a few $\mathrm{mN}$ only, rather than that of the complete gripping stage (Fig. 3). The compliant contact with the component surface compensates for the limited capture range of the hanging droplets. Thanks to this, the gripper merely requires coarse motion driving. The soft contact between capillary and component also enforces wetting and the repeatable formation of the capillary-die liquid bridge.

The complete capillary gripper system consists of four nominally identical capillaries arranged to span the four corners of the foil dies. This organization prevents uncompensated torques and consequent tilting of the dies upon pull-off of the gripper and enhances the stability of the operation.

\section{ANALYSIS OF THE CAPILLARY GRIPPER}

\section{Capillary Lifting Force}

A critical parameter for the gripping operation integral to the assembly process is the lifting force the capillary gripper can exert. The lifting force determines the limit weight of the components that can be handled and the accelerations they may be safely subjected to during handling.

To raise a component of mass $m_{\text {die }}$ from a supporting surface, the lifting capillary force $F_{C}$ needs to overcome the sum of the component weight $F_{G}=m_{\text {die }} g$ ( $g$ the acceleration of gravity), and the (non-capillary) adhesion force $F_{A}$ between component and resting surface. Since the accelerations imparted by our gripper on the component (a few $\mathrm{mm} / \mathrm{s}^{2}$ at maximum) are $\ll g$, the grasping condition is defined by:

$F_{C}>m_{d i e} g+F_{A} \quad(z=0)$

During component handling, the dynamic condition

$F_{C}>m_{d i e} a \quad(z>0)$

with $a$ is the cumulative component acceleration, enforces avoidance of break-up of the capillary-die liquid bridge. As described below, the lifting force exerted by the capillary gripper was measured experimentally, and the gripping operation was simultaneously video-recorded to track the evolution in time of the geometry of the capillary-die liquid bridge, defined in Fig. 4. A hydrostatic model was developed to frame the lifting force exerted by the open-ended capillary gripper.

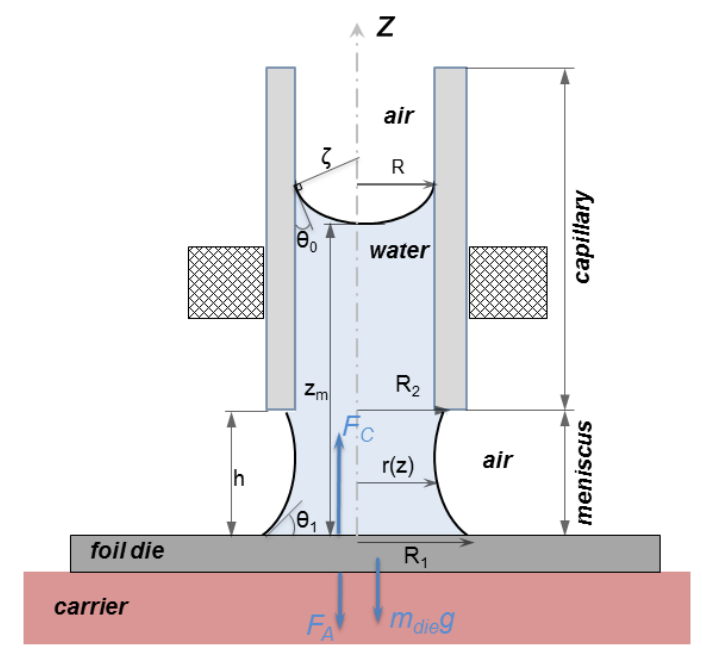



HERE TO EDIT) $<$

Fig. 4. Free-body diagram (not to scale) of a foil die resting onto a carrier and in contact with the gripper through the axisymmetric capillary-die liquid bridge. Relevant geometrical parameters of the capillary gripper are indicated.

\section{A. Lifting Force of the Capillary Gripper}

The lifting force exerted by metallic capillaries of different radii $R(0.41,0.83$ and $0.92 \mathrm{~mm}$, respectively) was measured experimentally through the setup sketched in Fig. 5a (and detailed in Appendix). A foil die was fixed to a precision electronic balance, and a sideview high-resolution camera in-situ recorded the shape of the capillary-die liquid bridge. A positioning base stage holding the shock insulator lock was used to bring a single water-filled capillary in contact with the top foil die surface. By pulling up the gripper at a speed $v_{p u}=10 \mu \mathrm{m} / \mathrm{s}$, the protruding liquid bridge was stretched vertically and the lifting force was measured as function of the optically-tracked gap $h$ between the bottom of the capillary and the foil surface.

For all capillaries tested, we recorded an initial increase of lifting force with increasing gap up to a maximum, after which the lifting force decreased with further increase of the gap, until pinch-off of the liquid bridge occurred (Fig. 5b). Wider capillaries exerted larger maximum lifting forces $F_{\max }$, which scaled with the square of their radius (i.e. meniscus contact area, Fig. 5c). Unnoticeable differences in the force versus gap curves where observed when varying $v_{p u}$ within the experimentally range of $1 \mu \mathrm{m} / \mathrm{s}$ up to $50 \mathrm{~mm} / \mathrm{s}$. The shape of a liquid bridge has a characteristic response rate proportional to the capillary velocity $v_{\text {cap }}=\gamma / \eta$, i.e. the ratio of surface tension $\gamma$ and viscosity $\eta$ of the liquid [28], amounting to $\approx 73 \mathrm{~m} / \mathrm{s}$ for a water/air interface at room temperature. Hence $v_{p u} \ll v_{c a p}$ in all cases, and the axial stretching was quasi-static.

a
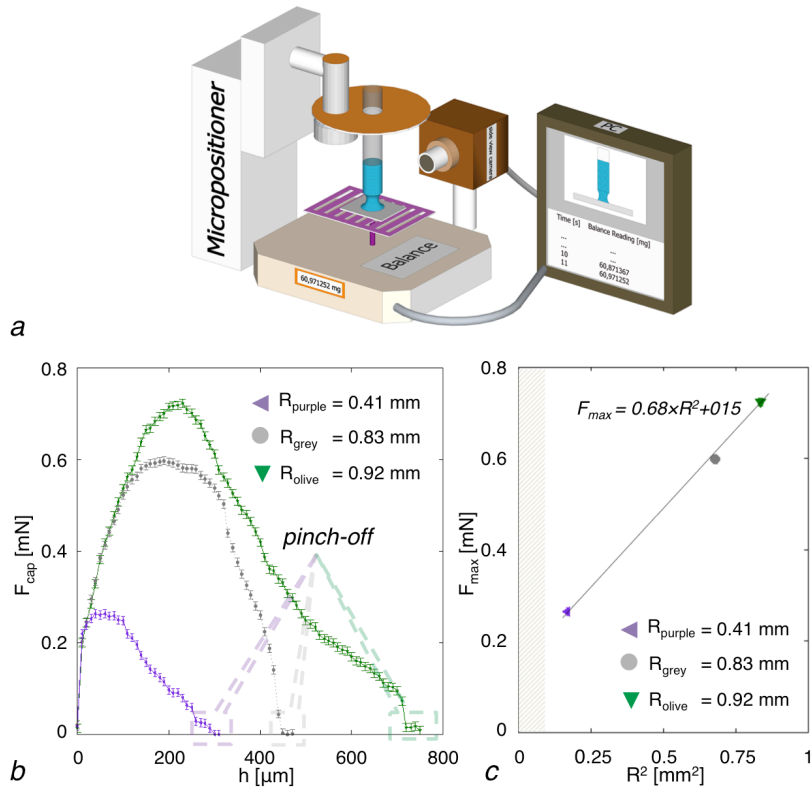

Fig. 5. (a) Schematic representation of the measurement setup for the lifting force exerted by a capillary gripper and the shape of the capillary-die liquid bridge, using an electronic balance and a sideview camera. (b) Lifting force generated by a capillary gripper as a function of gap height. (c) Maximum lifting force $F_{\max }$ versus $R^{2}$ (square radius of the capillary tube). Note that, since the neck radius of the liquid bridge is smaller than $R$ when $\mathrm{F}_{\max }$ is exerted, the regression does not extrapolate to $R^{2} \rightarrow 0$.
Relevant geometrical parameters of the liquid bridge were estimated from post-processing of the recorded frames. Fig. 6 shows experimental data for a capillary with $R$ of $0.83 \mathrm{~mm}$. Contrary to standard capillary grippers, in our case the volume of the capillary-die liquid bridge is not constant, and instead it increases with the gap (Fig. 6a). As the gap increases, gravity and negative Laplace pressure pull water from inside the capillary (of $16 \mu \mathrm{L}$ volume) into the bridge. The downward water flow is made possible by the additional water/air interface present at the open top end of the capillary. Moreover, during pull-off, the triple contact line of the meniscus remains pinned on the lower surface, and its contact area remains constant, as long as the contact angle $\theta_{i}$ is larger than its receding value $\theta_{i r}$ (Fig. 6b). Upon further stretching of the bridge, the bottom contact angle reaches $\theta_{\text {ir }}$ (Fig 6c), henceforth the contact line unpins and the contact radius $R_{l}$ is observed to decrease (Fig. 6d) with rather constant $\theta_{i}$ (Fig 6b). As the gap continues to increase, the liquid bridge thins down and finally pinches off at its neck, leaving a small droplet on the surface of the foil die after breakup. The rupture of the meniscus occurs at different elongations depending on the radius of the capillary (Fig. 5b). For the widest capillary $(R=0.92 \mathrm{~mm}$ ) forces up to $\sim 0.7 \mathrm{mN}$ (corresponding to $70 \mathrm{mg}$ of apparent weight) could be generated (Fig. 5b). By combining multiple capillaries into the gripper, the lifting force can be adapted to the user's needs.
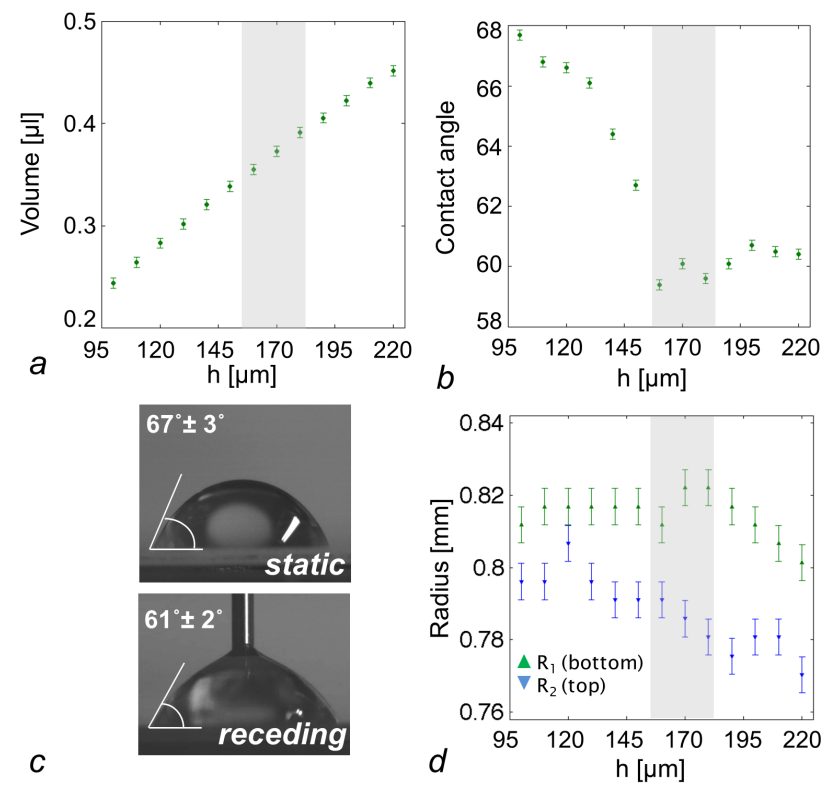

Fig. 6. (a) Volume $V$ of the liquid bridge versus gap height $h$. (b) Bottom contact angle $\theta_{t}$ of the liquid bridge as function of $h$. (c) Static $\theta$ and receding $\theta_{r}$ contact angles of water on PEN foil. (d) Contact radii of the liquid bridge: $R_{1}$ on the bottom foil die, $R_{2}$ on the rim of the capillary. Data in a), b) and d) obtained for the same metal capillary of radius $0.83 \mathrm{~mm}$ around the gap value (vertical grey band) yielding the maximal lifting force (shown in Fig. 5b).

\section{B. Analytic Model of the Capillary Gripper}

We derived an analytic model of the capillary gripper to obtain an accurate reconstruction of the geometrical profile of the liquid meniscus, and from that an estimate of the capillary lifting force was benchmarked with the experimental data. We particularly aimed at understanding and quantifying the specific role of the additional water/air interface of the top open end 
in the gripper performance (refer to the geometry of Fig. 4). With respect to prior models of liquid menisci bridging a vertically moving tip of finite width to an infinite planar substrate [29-30], our model assumes that the volume of the meniscus $V$ (which does not include that of water inside the capillary) is not a conserved quantity, rather it can increase as function of the gap (as observed in Fig. 6a).

The model is quasi-static, consistent with the dynamics of axial stretching described in the previous section. It reconstructs the geometry $r(z)$ of the capillary-die water meniscus, and from that the axial force applied by the gripper on the component, for a given gap height $h$. A force-versus-time profile of the gripping action can be obtained from the knowledge of the time sequence of the gap values. All parameters of the geometry could be obtained from the experiments, with the exception of the contact angle $\theta_{0}$ of the liquid on the inner capillary surface, which could not be visualized in real-time because opaque metallic capillaries were used in the reference experiment.

The profile $r(z)$ of the water meniscus is reconstructed from the axisymmetric Laplace equation [15]:

$$
\frac{\Delta p(z)}{\gamma}=-\frac{\frac{\partial^{2} r(z)}{\partial z^{2}}}{\left[1+\left(\frac{\partial r(z)}{\partial z}\right)^{2}\right]^{\frac{3}{2}}}+\frac{1}{r(z)\left(1+\left(\frac{\partial r(z)}{\partial z}\right)^{2}\right)^{\frac{1}{2}}}
$$

This second order, non-linear differential equation (3) can be solved by positing ( $'$ indicates derivative with respect to $z$ ):

$$
Y=\left(\begin{array}{l}
u=r(z) \\
v=r^{\prime}(z)
\end{array}\right) \rightarrow Y^{\prime}=\left(\begin{array}{l}
u^{\prime}(z) \\
v^{\prime}(z)
\end{array}\right)=\left(\begin{array}{c}
v \\
f(z, u, v)
\end{array}\right)
$$

thus obtaining $r(z)$ from the following system of first-order differential equations:

$$
\left\{\begin{array}{c}
u^{\prime}(z)=v \\
v^{\prime}(z)=-\frac{\Delta p(z)}{\gamma}\left(1+v^{2}\right)^{\frac{3}{2}}+\frac{1+v^{2}}{u}
\end{array}\right.
$$

complemented by the boundary conditions:

$$
\left\{\begin{array}{c}
\left.u\right|_{z=0}=R_{1} \\
\left.v\right|_{z=0}=-\frac{1}{\tan \theta_{1}}
\end{array}\right.
$$

and knowledge of $\Delta p(z)$, which can be obtained from:

$$
p_{\text {in }}(z)=p_{\text {atm }}+\Delta p_{\text {top }}+\rho g\left(z_{m}-z\right)
$$

where

$$
\Delta p_{\text {top }}=-\frac{2 \gamma}{\varsigma}=-\frac{2 \cos \theta_{0}}{R} \gamma
$$

is the pressure jump across the interface at the top open end of the capillary ( $\varsigma$ being its radius of curvature), $p_{\text {in }}(z)$ and $p_{\text {atm }}$ the pressure inside the capillary and in the atmosphere, respectively, and $z_{m}$ is the total height of the water column. Hence:

$\Delta p(z)=\rho g\left(z_{m}-z\right)-\frac{2 \cos \theta_{0}}{R} \gamma$

$$
\begin{aligned}
& =\left(\rho g z_{m}-\frac{2 \cos \theta_{0}}{R} \gamma\right)-\rho g z \\
& =f\left(\theta_{0}, \rho, V\right)-\rho g z
\end{aligned}
$$

The set of Eqs. 5 was solved for $r(z)$ through Matlab's ode 45 routine using Eq. 9 and the boundary conditions in Eq. 6 . The solution was accepted when the reconstructed value of $R_{2}=\left.r\right|_{z=h}$ coincided with its measured value within a relative error of $10^{-5}$. A self-consistent loop was implemented to determine the value of $z_{m}$ for each $h$ from the knowledge of $V$ and of the reconstructed volume of the meniscus. The total lifting force of the gripper was calculated as the sum of the terms dependent on Laplace pressure and surface tension [15]:

$$
F_{\text {tot }}(h)=\left.\pi R_{1}^{2}(h) \cdot \Delta p\right|_{z=0}+2 \pi R_{1}(h) \gamma \sin \theta_{1}(h)
$$

A shooting method [15] was used to solve this problem for each value of the gap in the experimental gripping sequence. The shooting variable was $\theta_{l}$ and $R_{l}$ respectively before and after $\theta_{1}$ reached its receding value (see Fig. 6b and Fig. 6d).
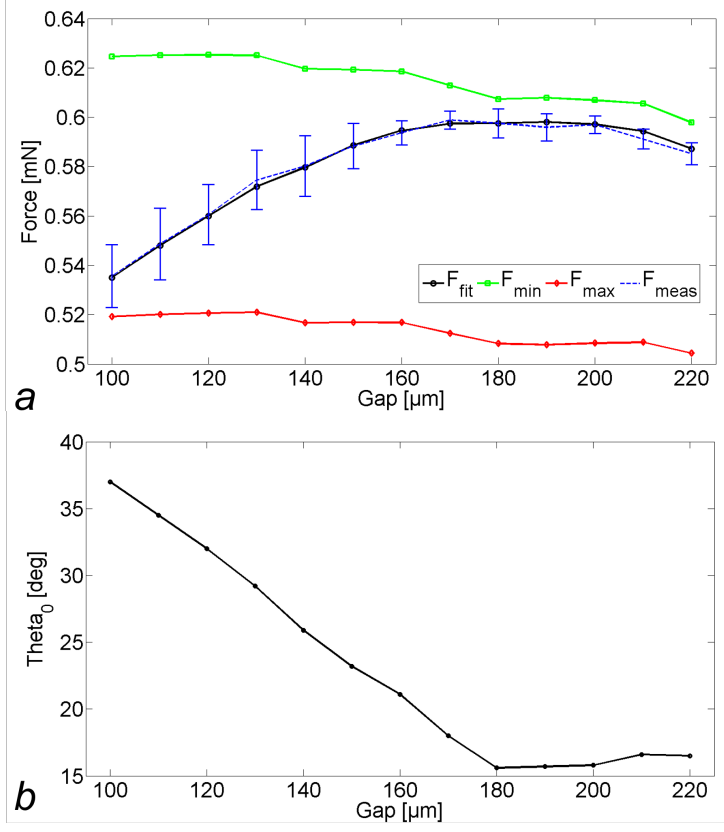

Fig. 7. Total capillary lifting force versus gap height for an open-ended capillary of $0.83 \mathrm{~mm}$ onto a PEN foil. $\mathrm{F}_{\text {meas }}$ is the experimentally measured value (mean and standard deviation). $F_{\min }$ and $F_{\max }$ are the forces predicted by the model for an interfacial profile at the open end of the capillary set by constant, minimum and maximum $\theta_{0}$ values of $10^{\circ}$ and $40^{\circ}$, respectively. $F_{f i t}$ is the total capillary force predicted using $\theta_{0}$ as fitting parameter of the model. The corresponding values of $\theta_{0}$ are represented in (b).

Fig. 7 provides a comparison between the predicted and the measured maximal gripping forces for the $0.83 \mathrm{~mm}$ radius capillary as a function of the gap $h$. In absence of experimental data, the water contact angle at the open end of the capillary $\theta_{0}(t)$ was used as free model parameter. Along with the force profile predicted by fitting $\theta_{0}$, in Fig. 7a for each value of $h$, we also show the numerical predictions of the force for two limiting and constant values of $\theta_{0}\left(10^{\circ}\right.$ and $40^{\circ}$, respectively $)$ that can encompass the measured values of the gripping force. The fitted values of $\theta_{0}(t)$, presented in Fig. 7b, are consistent with an interpretation based on the deformation (at constant 

HERE TO EDIT) $<$

position) and subsequent receding motion (at constant contact angle) of the water contact line at the top water/air interface, analogous to that of $\theta_{l}(t)$ shown in Fig. 6b. A numerical reconstruction of the meniscus profile is shown in Fig. 8.

The model can capture the physics of the experiment and quantitatively reproduce the experimental force data. The contact angle $\theta_{0}$ seems to play an important role in the modulation of the lifting capillary force. Its active use to control grasping and release operations, for instance through electrowetting, can be envisioned and is left for future investigations.

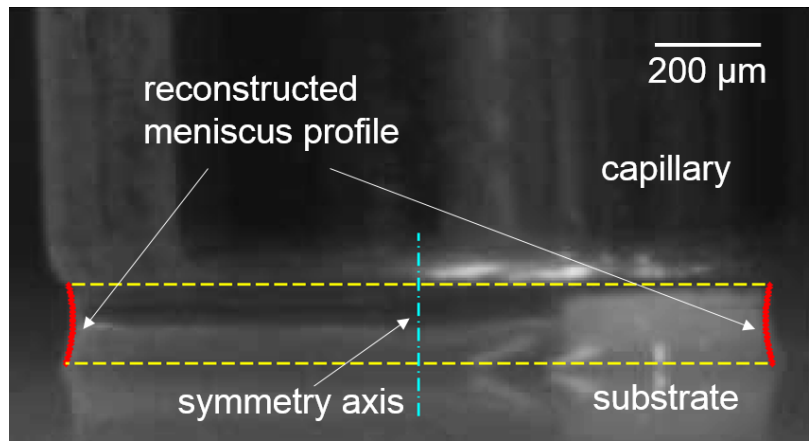

Fig. 8. Profile of capillary-die liquid bridge reconstructed by the model and superposed onto a video frame of the reference experiment $\left(h=180 \mu \mathrm{m}, \mathrm{R}=830 \mu \mathrm{m}, R_{l}=822 \mu \mathrm{m}, R_{2}=785 \mu \mathrm{m}, \theta_{0}=15.5^{\circ}\right)$.

\section{Adhesion of foil dies to supports}

The adhesion of components to their supporting surfaces, arising from possible interactions such as van der Waals forces, electrostatic and pressure imbalances during the lifting action, was found to play a non-negligible role in determining the required pick-up force $[9,31]$. We phenomenologically describe the cumulative adhesion force as $F_{A}=\beta \cdot l^{2}$ for a foil die of side length $l$, with $\beta$ a dimensional constant. Hence:

$$
F_{C}=m_{\text {die }} g+F_{A}=\alpha l^{2} g+\beta l^{2}=(\alpha g+\beta) l^{2}
$$

where $\alpha=0.36 \frac{\mathrm{mg}}{\mathrm{mm}^{2}}$ is the mass density of the PEN foil die (of constant thickness); and the size $l_{\max }$ of the largest foil die liftable by the maximum grasping force $F_{\max }$ (Fig. 5b) is:

$$
l_{\text {max }}=\sqrt{\frac{F_{\max }}{(\alpha g+\beta)}}
$$

To quantify the adhesion force, square-shaped foil dies ranging in sidelength from 5 up to $25 \mathrm{~mm}$ were gripped from three surfaces differing in the degree of porosity and roughness modulating the contact area with the die: soft cleanroom wipe made from polyester knit fabric (Super Polx®1200, Berkshire), paper (Black Label Zero, $80 \mathrm{~g} / \mathrm{m}^{2}$, Canon), and PEN foil. The gripper with four capillaries was used, and two sets of capillaries (radii of $0.92 \mathrm{~mm}$ and $0.41 \mathrm{~mm}$ ) were tested. For each surface type, the various foil dies were gripped five times at a pull-off velocity of $10 \mu \mathrm{m} / \mathrm{s}$. A correlation between maximum pulled load and type of support surface was observed (Fig. 9, and Fig. 15 in the Appendix). Larger foil dies could be gripped from porous wipes while not from the PEN foil. The adhesion force density for the three different surfaces was estimated to range from $3.5 \mu \mathrm{N} / \mathrm{mm}^{2}$ for cleanroom wipes to $13.6 \mu \mathrm{N} / \mathrm{mm}^{2}$ for PEN foil. Using porous, non-charged surfaces such as the wipes considerably reduced the required lifting forces and extended the gripping process window.

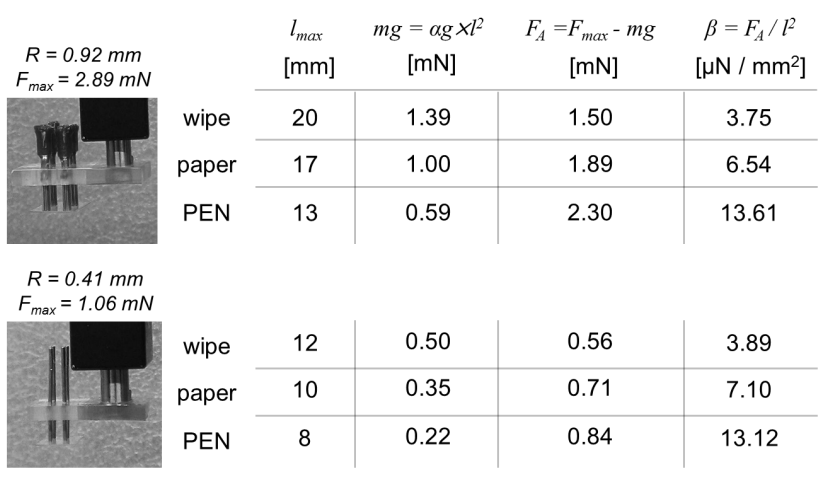

Fig. 9. Grasping of foil dies with the capillary grippers from different surface types and using two homogeneous sets of capillary tube (radii and maximum lifting forces indicated in the insets). From each surface type, a foil of each size was gripped five times with pull-off velocity of $10 \mu \mathrm{m} / \mathrm{s}$.

\section{Release and Self-Alignment}

The component release strategies we propose exploit the formation of the die-carrier liquid bridge to grasp a foil die suspended by the capillary-die bridge (see Fig. 2 and step 6 in Fig. 1). Not only is such bottom liquid bridge designed to prevail over the top capillary-die bridge: it is also the medium itself driving the capillary self-alignment of the component on its matching assembly site. The release of the component seamlessly coincides with the inception of its self-alignment.

The release condition is therefore:

$$
F_{C}<m_{\text {die }} g+F_{\text {Bot }}
$$

where $F_{B o t}$ is the axial capillary force exerted by the bottom liquid bridge. As previously shown, the capillary gripping force $F_{C}$ has a maximum value approximately proportional to the cross-section of the capillaries. Conversely, the axial force of the die-carrier liquid bridge increases monotonously in time, because the cross-section of the liquid bridge constantly and rapidly grows upon formation to ultimately encompass the full bottom surface of the die [23]. Since this surface is by design considerably larger than that of the capillaries, the grasping force exerted by the die-carrier bridge will rapidly become dominant, ensuring reliable component release.

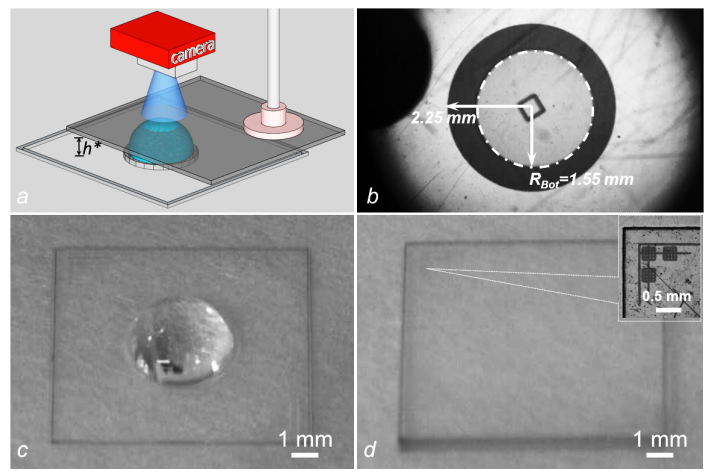

Fig. 10. (a) Sketch of a foil die brought in contact with a droplet confined in the center of an assembly site. (b) Top view of the foil/water interface created immediately upon contact of the foil die with the droplet. (c) $12.5 \mu \mathrm{l}$ water droplet pre-confined within the inner circular trench onto a carrier foil and (d) corresponding self-alignment of a shape-matching $10 \times 10 \mathrm{~mm}^{2}$ foil die. 

HERE TO EDIT) $<$

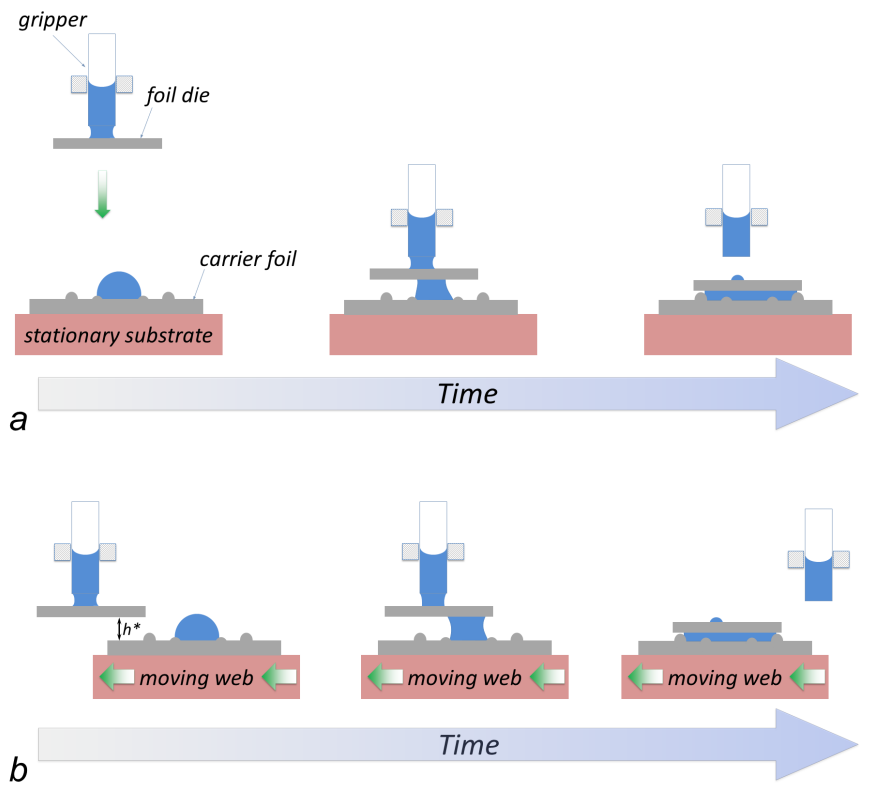

Fig. 11. Schematic sideview of the two alternative, passive capillary strategies for component release: 1) release by vertical capillary forces on stationary carrier, and 2) dynamic shear release by lateral capillary forces on moving web.

We performed experiments using $10 \times 10 \mathrm{~mm}^{2}$ PEN foil dies and shape-matching assembly sites to assess the capillary release performance. $12.5 \mu \mathrm{l}$ water drops were pre-deposited within inner circular trenches of $2.25 \mathrm{~mm}$ radius (Fig. 10c). Such tight confinement yields $\sim 1 \mathrm{~mm}$ high droplets, an immediate water overflow beyond the circular trench upon contact with the foil die [33], and ensures optimal assembly performance [25] (Fig. 10d). The foil die held by a micropositioner was coarsely superposed onto the assembly site and then lowered to precisely contact the confined droplet and form a diesubstrate liquid bridge at a gap height $h^{*}$ of $0.8 \mathrm{~mm}$ (Fig. 10a). As a result, a circular water/PEN interface with radius $R_{b o t}=$ $1.55 \mathrm{~mm}$ formed instantaneously on the bottom surface of the foil die (Fig. 10b), yielding an estimated initial $F_{B o t}$ of $\sim 1.8$ $\mathrm{mN}$, which grew up to $\sim 20 \mathrm{mN}$ when after few milliseconds the meniscus spread to cover the entire surface. From the above results we could tune the force involved in the carrierdie liquid bridge with respect to the capillary-die liquid bridge to optimize the both pick-up and release of the foil dies.

To confirm our modeling of all relevant aspects of the process, we set $10 \times 10 \mathrm{~mm}^{2}$ foil dies (weight of $0.35 \mathrm{mN}$ ) on a cleanroom wipe (adhesion force of $0.7 \mathrm{mN}$ ). The minimal force required by the gripper to pick up the foil dies was thus estimated to be $\sim 1.05 \mathrm{mN}$. Four glass capillaries fabricated from Pasteur pipettes (Wu Mainz, Germany; radius of 0.65 $\mathrm{mm}$ ) were chosen for the gripper, resulting in a capillary gripping force around $\sim 1.74 \mathrm{mN}$. Next the foil dies were prealigned with a corresponding assembly site, lowered down to $\sim 0.8 \mathrm{~mm}$ above the carrier substrate and brought into contact with the droplet. Passive release and ensuing self-alignment of the foil dies was then observed as expected, given the previously estimated value of $F_{\text {Bot }}(1.8 \mathrm{mN})$. The experiments were repeated over 15 times with no assembly failure (Fig. 10d).

\section{Release strategies}

\section{A. Vertical Component Release}

In conventional pick-and-place assembly [23], components are assembled onto assembly sites pre-defined over stationary assembly substrates. Such vertical release strategy was hereby mimicked and studied, as described in the previous sections, by keeping a pre-patterned foil substrate fixed while the capillary gripper was grasping, moving and releasing the foil die (Fig. 1 and Fig 11a).

\section{B. Horizontal Component release}

The fast and reliable release dynamics afforded by the diecarrier liquid bridge prompted an alternative release strategy based on a shearing capillary action. This novel, distinctive release technique is compatible with system integration by capillary self-alignment onto moving substrates, i.e. a continuously moving, automatic assembly line (Fig. 11b). The process proceeds similarly to that of the vertical die release as far as substrate patterning, droplet dispensing and component grasping are concerned (steps 1-5 of Fig. 1). Then, the foil die is suspended at a specific height $h^{*}$ lower than the bottom droplet's height. In this way, the droplet moving with the substrate will intercept the foil die. Upon contact between the foil die and the droplet confined on the assembly foil, the die-carrier bridge forms within milliseconds and the die is automatically grasped. Given the relatively high speed of $\approx 50 \mathrm{~m} / \mathrm{s}$ [33] of the self-alignment process compared to the translational speed of the substrate of up to $80 \mathrm{~mm} / \mathrm{s}$, the die-carrier liquid bridge has enough time to form and release the die from the gripper during the transient motion.

Such dynamic shear release technique is minimalistic, autonomous, non-triggered and programmable, thus greatly beneficial for the scalability and throughput of the integration process. Since the release merely relies on the contact of the bottom moving droplet with the suspended foil die, the foil dies can be held above the web for an arbitrary period of time ready for assembly. Release will only be initiated once a droplet slides underneath the suspended foil dies. Provided that the thickness of the components is smaller than the initial height of the confined droplets, the passive grasping and release process is not frustrated by components already assembled on the substrate, and it can be programmed to smoothly populate the entire substrate. By the same token, multiple grippers, each holding a component, could be set simultaneously along the main web axis to pipeline the assembly process.

\section{DEMONSTRATOR}

To provide a comprehensive verification, the aforementioned all-capillary assembly processes with the two release methods were tested to self-align 11 dummy $10 \times 10 \mathrm{~mm}^{2}$ foil dies, each weighting $0.35 \mathrm{mN}$ and marked with a letter spelling "Holst Centre". The system used for the demonstration consisted of a moving stage, the capillary gripper, the foil dies, the assembly substrate with scribed trenches and confined water droplets, and a water reservoir. The foil dies were placed by hand onto a wipe at a pitch of $15 \mathrm{~mm}$ in the same order as they would be 
assembled on the carrier substrate. The capillary gripper with the shock insulator lock was fixed to a z-axis stage so that it could be moved up during stage translations and down during grapping and releasing. The gripper was realized with 4 glass capillaries. An overview of the platform is shown in Fig. 12.

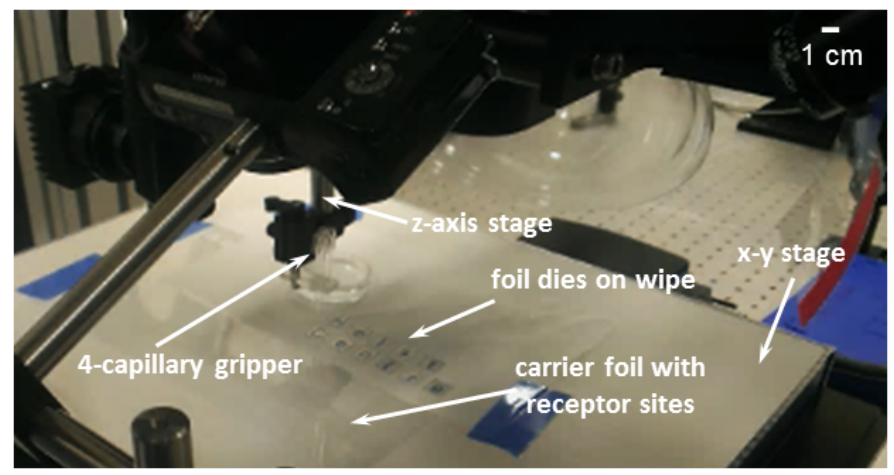

Fig. 12. Platform used to demonstrate the assembly processes. The capillary gripper is connected through the shock insulator to the z-stage. Foil dies are placed onto a cleanroom wipe. A water reservoir is provided at the back to fill the capillaries. A carrier assembly foil with assembly sites is placed in the front. $1 \mathrm{~mm}$ high water droplets are confined in the circular trenches. A positioning stage is used to perform all lateral movements.

Two cameras recorded the overview and side (close-up) view of the assembly process. A high-resolution microscope camera was used to inspect alignment precision after foil die assembly on the carrier foil. The full video of the assembly process (provided in Supplementary Information) demonstrates how the strategy was used to accurately and reliably pick and place the foil dies using the vertical release strategy. Excerpts are given in Fig. 13. Fig 13a shows a skewed foil die held over the assembly site by the capillary gripper. The foil die is then lowered to $\sim 0.8 \mathrm{~mm}$ above the substrate to come into contact with the droplet (Fig. 13b), then the gripper is raised by $1 \mathrm{~cm}$ (Fig 13c) while the die is released from the gripper through the carrier-die liquid bridge and self-aligns with respect to the outer scribed trenches (Fig. 13d). The accu-
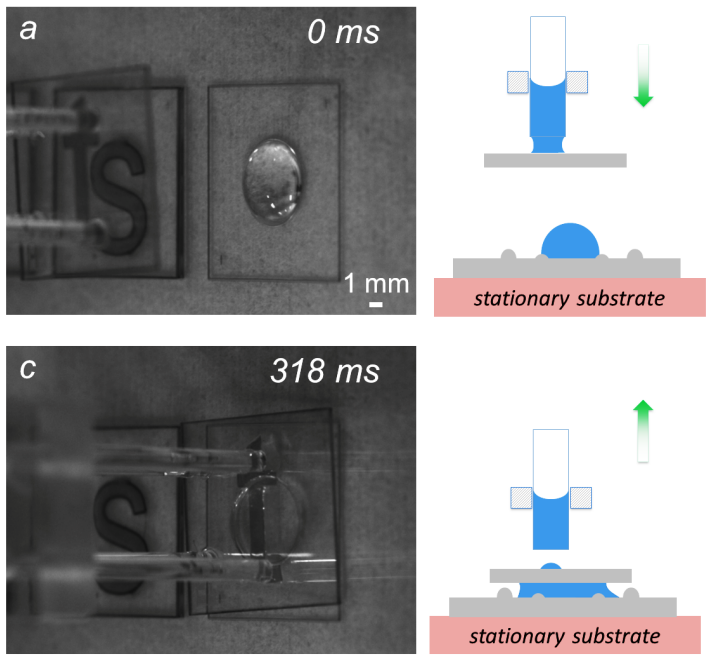
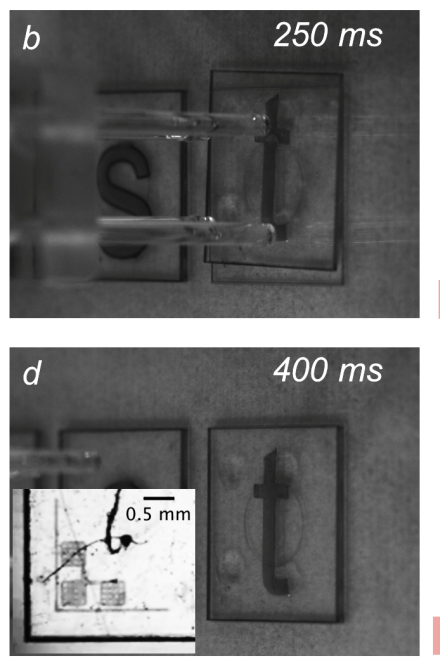

racy of the alignment, quantified through the laser-scribed markers (inset of Fig. 13d), was $\sim 20 \mu \mathrm{m}$, a figure consistent with the accuracy of the laser scribing process [23]. The procedure was successfully repeated for all foil dies.

The shear release strategy was demonstrated analogously, the key difference being that the assembly on a moving web was simulated by picking up each foil die with the gripper and translating the gripper in front of the line of the preset assembly sites, as shown in Fig. 14a. The capillary gripper held the foil dies approximately $0.8 \mathrm{~mm}$ above the assembly substrate while the stage moved the assembly sites at a predefined velocity of $80 \mathrm{~mm} / \mathrm{s}$. In Fig. 14 the letter ' $\mathrm{t}$ ' is assembled to spell the word 'Holst'. Fig. 14a-c show how the suspended foil die passes above the previously assembled foil dies, until the die comes into contact at full velocity with the droplet of the first available assembly site. Upon contact of the foil die with the pre-confined droplet, the die-carrier liquid bridge is seen to immediately form and release the foil die from the grasp of the capillary gripper [Fig. 14d]. The released foil die, now connected through the liquid bridge to the moving carrier substrate, slightly overshoots its corresponding assembly site due to its inertia [Fig. 14d-h]. The capillary restoring force of the laterally stretched meniscus in turn exerts a shear stress that pulls the foil die backwards. The die undergoes self-alignment and finally aligns with high precision $(\sim 20 \mu \mathrm{m}$, inset in Fig. $14 \mathrm{~h}$ ) onto its assembly site. A video recording of the full process can be found in the 'Supplementary Information'.

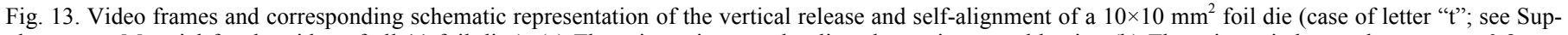

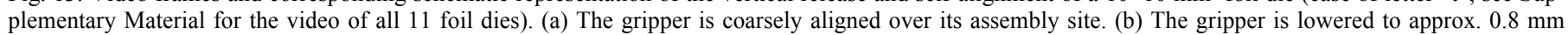

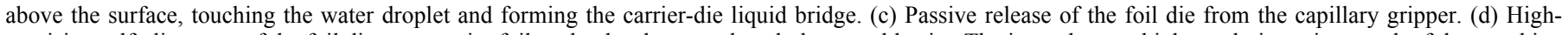

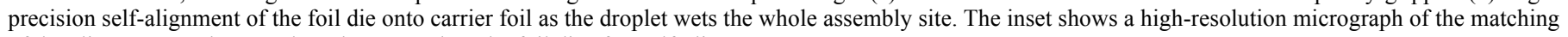
of the alignment markers on the substrate and on the foil die after self-alignment. 

HERE TO EDIT) $<$
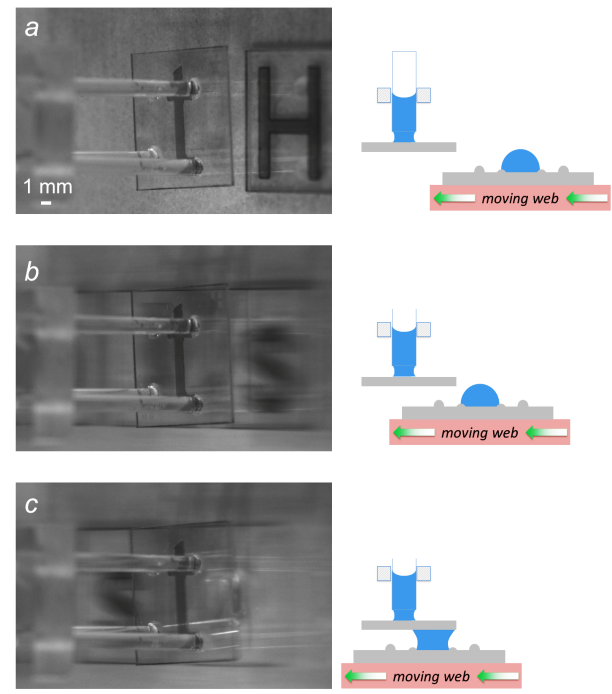

Fig. 14. Overviews, side views and corresponding schematic representations of shear capillary release and self-alignment process on a moving web of a $10 \times 10$ $\mathrm{mm}^{2}$ foil die (case of letter "t", see Supplementary Material for the video of all 11 foil dies). (a) Foil die lowered by the gripper to the preset height $h^{*}$. (b-c) The suspended foil die slides over the assembled dies towards the first available water droplet confined within the inner circular trench. (d) The foil die comes into contact with water droplet and the carrier-die liquid bridge is formed. (e) The carrier-die liquid bridge takes over the die from the capillary-die liquid bridges. (fh) Video frames from high-speed recording of the self-alignment of foil die onto the moving assembly substrate. The inset in (h) shows the matching of the alignment markers of the foil die and of the assembly site after self-alignment.

\section{CONCLUSION}

We introduced a new technique for cost-effective and highprecision assembly of components onto substrates. The fluidic assembly process exploits competing liquid bridges to combine capillary grasping, handling, passive release and selfalignment of components onto pre-patterned substrates within a seamless robotic procedure. Measured gripping forces were benchmarked through a hydrostatic model of the axisymmetric capillary gripper with both ends open. The model accounts for the evolving curvature of the profile of the liquid meniscus, and shows that the additional liquid/air interface present at the top open end of the gripper has significant impact on the grasping process. The adhesion forces of the components to various support surfaces were quantified, and the size, number and lifting force of capillaries were optimized.

A novel passive component release strategy based on capillary shear forces and pre-patterned moving substrates was proposed and validated through the autonomous assembly of foils dies. The capillary grasping and release techniques presented are expected to be beneficial for the assembly of components of a wide range of sizes [19-20].

Finally, the dynamic shear release strategy hereby demonstrated enables system integration by capillary self-alignment of dies onto continuously moving substrates. Prospects of a parallelized, autonomous and continuous roll-to-roll assembly line make it an advantageous mass production approach.

\section{REFERENCES}

A. I. L. Atzori, and G. Morabito, "The Internet of Things: A survey," Computer Networks, vol. 54, no. 15, pp. 2787-2805, 2010.

G. K. Fedder, R. T. Howe, L. Tsu-Jae King, and E. P. Quevy, "Technologies for Cofabricating MEMS and Electronics," Proceedings of the IEEE, vol. 96, pp. 306-322, 2008.
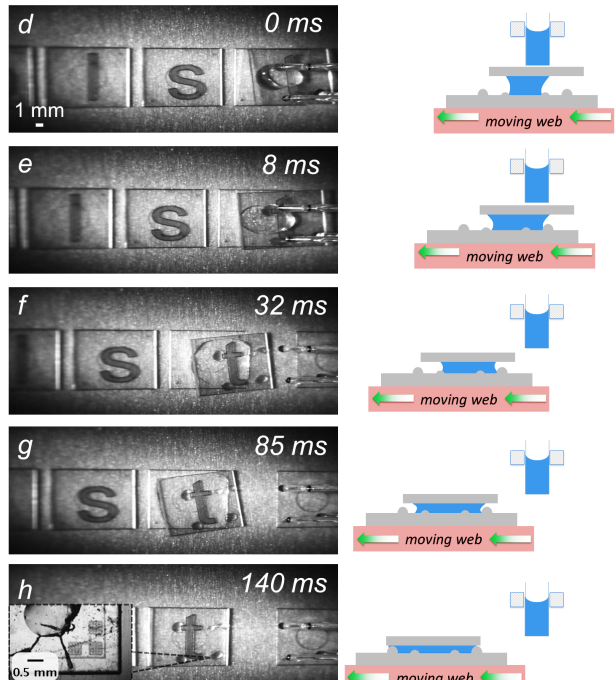

H
M. Lapisa, G. Stemme, and F. Niklaus, "Wafer-Level Heterogeneous Integration for MOEMS, MEMS, and NEMS," Selected Topics in Quantum Electronics, IEEE Journal of, vol. 17, pp. 629-644, 2011.

M. V. F. Alimenti, P. Mezzanotte et al., "Low-cost assembly of UHF RFID chips and flexible substrate antennas by magnetic coupling approach," MTT-S International, pp. 1532-1535, 2010. J. Chung, Z. Wei, T. J. Hatch, and H. O. Jacobs, "Programmable reconfigurable self-assembly: parallel heterogeneous integration of chip-scale components on planar and nonplanar surfaces," Microelectromechanical Systems, Journal of, vol. 15, pp. 457-464, 2006.

G. Fantoni and M. Porta, "A Critical Review of Releasing Strategies in Microparts Handling," in Micro-Assembly Technologies and Applications. vol. 260, S. Ratchev and S. Koelemeijer, Eds., ed: Springer US, 2008, pp. 223-234.

H. Van Brussel, J. Peirs, D. Reynaerts, A. Delchambre, G. Reinhart, N. Roth, et al., "Assembly of Microsystems," CIRP Annals - Manufacturing Technology, vol. 49, pp. 451-472, 2000.

F. B. G. Fantoni, "Design of a novel electrostatic gripper," Journal for Manufacturing Science and Production, vol. 6, pp. 163-179, 2004.

B. Bhushan, "Adhesion and stiction: Mechanisms, measurement techniques, and methods for reduction," Journal of Vacuum Science \& Technology B: Microelectronics and Nanometer Structures, vol. 21, pp. 2262-2296, 2003.

A. Vasudev and Z. Jiang, "A capillary microgripper based on electrowetting," Appl. Phys. Lett., vol. 93, pp. 103503-103503-3, 2008.

B. Lopez-Walle, M. Gauthier, and N. Chaillet, "Principle of a Submerged Freeze Gripper for Microassembly," Robotics, IEEE Transactions on, vol. 24, pp. 897-902, 2008.

F. Arai, T. Sakami, K. Yoshikawa, H. Maruyama, and T. Fukuda, "Synchronized laser micromanipulation of microtools for assembly of microbeads and indirect manipulation of microbe," in Intelligent Robots and Systems, 2003. (IROS 2003). Proceedings. 2003 IEEE/RSJ International Conference on, 2003, pp. 2121-2126 vol.3. V. Vandaele, P. Lambert, and A. Delchambre, "Non-contact handling in microassembly: Acoustical levitation," Precision Engineering, vol. 29, pp. 491-505, 2005.

T. O. Anders Petterson, Darwin G. Caldwell, Steven Davis, John O. Gray, Tony J. Dodd, "A Bernoulli principle gripper for handling of planar and 3D (food) products," Industrial Robot: An International Journal, vol. 37, pp. 518 - 526, 2010. 
[15] P. Lambert and A. Delchambre, "Parameters Ruling Capillary Forces at the Submillimetric Scale," Langmuir, vol. 21, pp. 95379543, 2005/10/01 2005.

[16] K. F. Böhringer, R. S. Fearing, and K. Y. Goldberg, "Microassembly," in Handbook of Industrial Robotics, ed: John Wiley \& Sons, Inc., 2007, pp. 1045-1066.

[17] P. Lambert, F. Seigneur, S. Koelemeijer, and J. Jacot. "A case study of surface tension gripping: the watch bearing." Journal of Micromechanics and Microengineering, vol. 16, 1267, 2006.

[18] G. Fantoni, H. N. Hansen, and M. Santochi, "A new capillary gripper for mini and micro parts," CIRP Annals - Manufacturing Technology, vol. 62, pp. 17-20, 2013.

[19] K. J. Obata, T. Motokado, S. Saito, and K. Takahashi, "A scheme for micro-manipulation based on capillary force," J. Fluid Mech., vol. 498, pp. 113-121, 2004.

[20] O. Fuchiwaki and K. Kumagai, "Development of wet tweezers based on capillary force for complex-shaped and heterogeneous micro-assembly," in Intelligent Robots and Systems (IROS), 2013 IEEE/RSJ International Conference on, 2013, pp. 1003-1009.

[21] F. Biganzoli, I. Fassi, and C. Pagano, "Development of a gripping system based on capillary force," in Assembly and Task Planning: From Nano to Macro Assembly and Manufacturing, 2005. (ISATP 2005). The 6th IEEE International Symposium on, 2005, pp. 36-40.

[22] C. Bark, T. Binnenbose, G. Vogele, T. Weisener, and M. Widmann, "Gripping with low viscosity fluids," in Micro Electro Mechanical Systems, 1998. MEMS 98. Proceedings., The Eleventh Annual International Workshop on, 1998, pp. 301-305.

[23] G. Arutinov, M. Mastrangeli, E. C. P. Smits, G. van Heck, J. M. J. den Toonder, and A. Dietzel, "Foil-to-Foil System Integration through Capillary Self-Alignment Directed by Laser Patterning," Journal of Microelectromechanical Systems,

DOI: 10.1109/JMEMS.2014.2321013 .

[24] V. Sariola, Q. Zhou, and H. Koivo, "Hybrid microhandling: a unified view of robotic handling and self-assembly," Journal of Micro-Nano Mechatronics, vol. 4, pp. 5-16, 2008/11/01 2008.

[25] G. Arutinov, M. Mastrangeli, E. C. P. Smits, H. F. M. Schoo, J. Brugger, and A. Dietzel, "Dynamics of capillary self-alignment for mesoscopic foil devices," Appl. Phys. Lett., vol. 102, pp. 144101144101-5, 2013.

[26] G. Arutinov, E. C. P. Smits, M. Mastrangeli, G. v. Heck, J. v. d. Brand, H. F. M. Schoo, et al., "Capillary self-alignment of mesoscopic foil components for sensor-systems-in-foil," Journal of Micromechanics and Microengineering, vol. 22, p. 115022, 2012.

[27] H. Aoyama, S. Hiraiwa, F. Iwata, J. Fukaya, and A. Sasaki, "Miniature robot with micro capillary capturing probe," in Micro Machine and Human Science, 1995. MHS '95., Proceedings of the Sixth International Symposium on, 1995, pp. 173-178.

[28] J. Eggers, "Nonlinear dynamics and breakup of free-surface flows", Reviews of Modern Physics, vol. 69, pp.865-929, 1997.

[29] J. Qian and H. Gao. "Scaling effects of wet adhesion in biological attachment systems." Acta biomaterialia ,vol 2, pp. 51-58, 2006.

[30] Y. Su, B. Ji, Y. Huang, K Hwang, "Effects of contact shape on biological wet adhesion." Journal of Materials Science, vol. 42, pp. 8885-8893, 2007.

[31] M. Savia, H. N. Koivo, and Q. Zhou, "Evaluation of adhesion forces between arbitrary objects for micromanipulation," Journal of Micromechatronics, vol. 3, pp. 221-238, 2006.

[32] N. Boufercha, J. Sägebarth, M. Burgard, N. Othman, D. Schlenker, W. Schäfer, et al., "Fluidassem - A New Method of Fluidic-Based Assembly with Surface Tension," in Micro-Assembly Technologies and Applications. vol. 260, S. Ratchev and S. Koelemeijer, Eds., ed: Springer US, 2008, pp. 149-159.

[33] G. Arutinov, E. C. Smits, P. Albert, P. Lambert, M. Mastrangeli, "In-plane mode dynamics of capillary self-alignment." Langmuir vol. 30, pp. 13092-13102, 2014.

\section{Appendix: Materials and Methods}

\section{Foil dies and carriers}

Polyethylene Naphthalate (PEN, Teonex ${ }^{\circledR}$ Q65FA, Teijin DuPont) was used as base material. $125 \mu \mathrm{m}$-thick foil sub- strates and $250 \mu \mathrm{m}$-thick foil dies were respectively patterned and ablated using a Coherent AVIA $355 \mathrm{~nm}, 25 \mathrm{~ns} \mathrm{Nd}: \mathrm{YAG}$ laser source in combination with a galvano-scanner. Static and receding water contact angles on the foil surfaces were measured through the static and contraction sessile drop methods, respectively (EasyDrop, KRÜSS).

\section{Capillary gripper}

The gripper featured cylindrical tubes fixed onto a lightweight 3-mm poly methyl methacrylate (PMMA) supporting plate, attached to the positioning stage through a onedirectional locking mechanism (Fig. 3). The capillaries were fabricated from precision, stainless steel dispense tips (Nordson EDF) consisting of an open metal tube attached to a polypropylene leur lock connector.

\section{Measurement setup}

The 3-axis motion system was made of an in-plane positioning stage (Newport MM4006) combined with a vertical motion stage (Xenus Plus, Copley Controls), both providing $1 \mu \mathrm{m}$-resolved displacements. The system was used to bring the water-filled capillary tube in contact with the surface of the foil anchored to the plate of the high-precision balance (XS105, Mettler Toledo). Retraction of the gripper was controlled through a stepper motor with no hysteresis in a velocity range of $1 \leq V \leq 20 \times 10^{3} \mu \mathrm{m} / \mathrm{s}$. Upon gripper pull-up, the evolution of the capillary bridge geometry as a function of the gap was monitored through a synchronized sideview camera (Stingray, Allied Vision Tech.) with resolution of $5 \mu \mathrm{m}$ per pixel. Quantities entering into Eq. 2 and 3, such as $\theta_{l}, R_{l}$ and were estimated by image post-processing. Low contrast of acquired images led to relatively small systematic errors (Fig. $6 \mathrm{~b}$ and $6 \mathrm{~d})$.

\section{Success chart}
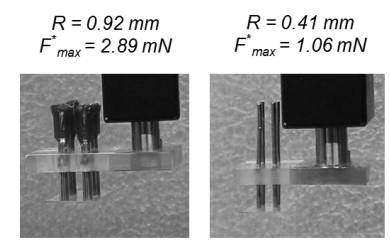

Side length
[mm]
25
24
23
22
21
20
19
18
17
16
15
14
13
12
11
10
9
8
7
6
5
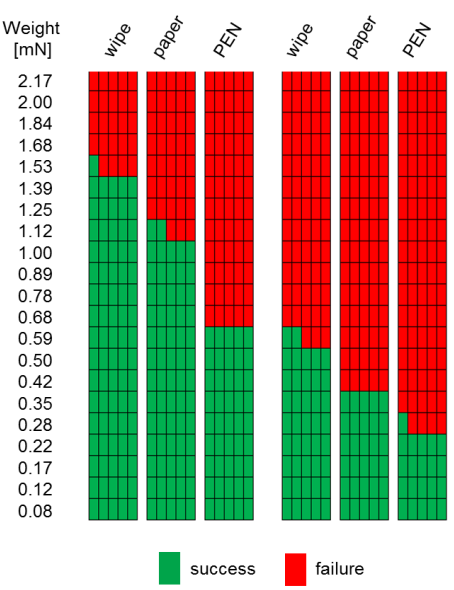

Fig. 15. Success chart for lifting of foil dies from different surfaces through the two capillary grippers. From each surface type, one foil of each size was gripped five times with pull-off velocity of $10 \mu \mathrm{m} / \mathrm{s}$. 